\title{
XRPD as a tool for the study of pigment-binder interactions: from metal formates to long-chain carboxylates
}

\author{
S. Švarcová ${ }^{1 *}$, E. Kočí ${ }^{1}$, P. Bezdička ${ }^{1}$, S. Garrappa ${ }^{1}$, J. Plocek ${ }^{1}$, R. Barranikov ${ }^{1}$, L. Kobera ${ }^{2}$ \\ ${ }^{1}$ Institute of Inorganic Chemistry of the Czech Academy of Sciences, ALMA Laboratory, Husinec-Řež 1001, 25068 Husinec- $\check{R} e z ̌$, \\ Czech Republic, \\ ${ }^{2}$ Institute of Macromolecular Chemistry of the Czech Academy of Sciences, Heyrovského nám. 2, 16206 Praha 6, Czech Republic,
} svarcova@iic.cas.cz

Pigment-binder interactions occurring in paint layers of artworks can either contribute to the stability of paint films as well as they can cause their degradation, seriously affecting the appearance and stability of paintings. Depending on intrinsic (i.e., composition of pigments and/or binders, presence of additives etc.) or extrinsic factors (i.e., relative humidity, temperature, conservation treatment etc.), formation of metal carboxylates can fulfil both aspects. Metal carboxylates result from reactions between metal-based pigments with carboxylic acids originating from a fatty medium. In paint layers, the reactive metal pigments are represented especially by leadbased pigments (e.g., lead white, red lead, lead-tin yellows etc.) or zinc white while fatty binder usually means drying oils (e.g., linseed oil, poppy-seed oil, walnut oil etc.) or even egg yolk. [1,2] On one hand, these pigments accelerate drying of paint layers but, on the other hand, the neo-formed crystalline phases tend to aggregate, resulting in formation of inclusions, protrusions, crusts, blisters or efflorescence. Moreover, reacting with atmospheric gases and pollutants, metal carboxylates can induce the cascade degradation, often accompanied by changes in the tonality of paint layers. Understanding the processes in paint layers is essential for the development of suitable conservation strategies which are necessary to prevent these types of degradation. Since the paintings comprise complicated multi-layered systems in which each particular layer consists of numerous inorganic and organic components, the experimental studies performed on simplified model paint systems are reasonable for identification and description of the pigmentbinder interactions. Complementing vibrational spectroscopies, XRPD represents and effective tool for detection of crystalline phases, especially if unexpected product such as metal formates occur. [1] On the other hand, usually robust XRPD meets certain limits in case of metal carboxylates with undetermined crystal structure. [3] Finally, XRPD can be also beneficial for the description of metal carboxylates adopting ionomer structures. Within the contribution, the advantages and limits of XRPD for study of pigment-binder interactions in paint layers will be discussed.

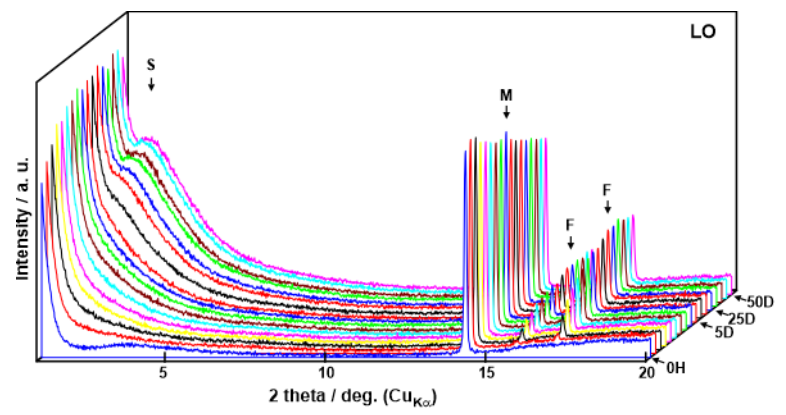

Figure 1. Time-dependent XRD patterns of model paint consisting of minium and linseed oil (LO). The XRD patterns between 0 hours $(0 \mathrm{H})$ and 5 days $(5 \mathrm{D})$ were collected every 12 hours and, further on, every week. The detected phases: $\mathrm{F}$ - lead formate, $\mathrm{Pb}(\mathrm{HCOO})_{2} ; \mathrm{M}-$ minium $-\mathrm{Pb}_{3} \mathrm{O}_{4} ; \mathrm{S}-\mathrm{Pb}$-soap/ionomer.

[1] Švarcová, S., Kočí, E., Bezdička, P., Garrappa, S., Kobera, L., Plocek, J., et al. (2020). Dalton Trans. 49, 5044.

[2] Švarcová, S., Kočí, E., Plocek, J., Zhankina, A., Hradilová, J., Bezdička, P. (2019). J. Cul. Herit. $38,8$.

[3] Kočí, E., Rohlíček, J., Kobera, L., Plocek, J., Švarcová, S., Bezdička, P. (2019). Dalton Trans. 48, 12531.

Keywords: metal carboxylates; pigment-binder interactions; paintings; XRPD

The study was supported by the Czech Academy of Sciences in the frame of the programme Strategy AV21 no. 23 - City as a Laboratory of Change, Historical Heritage and Place for Safe and Quality Life.

Acta Cryst. (2021), A77, C504 